Research Article

\title{
Analytical Consideration of Growth in Population via Homological Invariant in Algebraic Topology
}

\author{
Lewis Brew $\mathbb{D}^{1},{ }^{1}$ William Obeng-Denteh $\mathbb{D}^{2},{ }^{2}$ and Fred Asante-Mensa ${ }^{2}$ \\ ${ }^{1}$ Department of Mathematical Sciences, University of Mines and Technology, Tarkwa, Ghana \\ ${ }^{2}$ Department of Mathematical Sciences, Kwame Nkrumah University of Science and Technology, Kumasi, Ghana
}

Correspondence should be addressed to Lewis Brew; lbrew@umat.edu.gh

Received 9 April 2020; Accepted 28 April 2020; Published 13 May 2020

Academic Editor: Basil K. Papadopoulos

Copyright (c) 2020 Lewis Brew et al. This is an open access article distributed under the Creative Commons Attribution License, which permits unrestricted use, distribution, and reproduction in any medium, provided the original work is properly cited.

This paper presents an abstract approach of analysing population growth in the field of algebraic topology using the tools of homology theory. For a topological space $X$ and any point $v^{n} \in X$, where $v^{n}$ is the $n$-dimensional surface, the group $\eta=\left(X, v^{n}\right)$ is called population of the space $X$. The increasing sequence from $v_{i}^{n} \in X$ to $v_{j}^{n} \in X$ for $i<j$ provides the bases for the population growth. A growth in population $\eta=\left(X, v^{n}\right)$ occurs if $v_{i}^{n}<v_{j}^{n}$ for all $v_{i}^{n} \in X$ and $v_{j}^{n} \in X$. This is described by the homological invariant $H\left(\eta_{k}\right)=1$. The aim of this paper is to construct the homological invariant $H\left(\eta_{k}\right)$ and use $H\left(\eta_{k}\right)=1$ to analyse the growth of the population. This approach is based on topological properties such as connectivity and continuity. The paper made extensive use of homological invariant in presenting important information about the population growth. The most significant feature of this method is its simplicity in analysing population growth using only algebraic category and transformations.

\section{Introduction}

Algebraic topology which provides computable property for identifying topological properties has gained importance and popularity in many diverse fields of mathematics and other branches of science. Though the subject algebraic topology is a relatively new field of mathematics, its contribution to the study of the dynamics in both metric and topological spaces cannot be overruled. For instance, in the study of the components of a space, it provides a lot of information about the number of the components and how they are linked together in the space. Not only does the information gained helps us to understudy both topological and geometric properties of the space, but also provides a method of reducing topological problems and continuous maps into algebraic problems, thereby making it appropriate to determine some homological invariants. The essence of the homological invariant in this study is that it does not change under the deformations for the same property. Rather, it assigns a particular number to a specific property under study. Population growth also remains to grow no matter how wide the population growth varies. This is assumed as a homological invariant under growth deformation. In this paper, a specific value is assigned to the homological invariant to represent the population growth. Though different methods such as statistical analysis and differential equation models have been developed and used in studying population growth in many research works, many are yet to be developed, which perhaps, may contribute meaningfully to the existing knowledge. Indeed, to study the growth of population of a country, one needs to be equipped with all the necessary information regarding the population of the country, and the approach to collect all this information is sometimes quite arduous in the field of statistics and differential equations. Research on population growth and its implication in Walker [1] revealed that nations with the fastest growing populations tend to rank high on global indices of hunger, poverty, and environmental degradation. Guney [2] also made it clear in his work that while population growth in developing countries affects sustainable development negatively, the population growth in developed countries affects sustainable growth positively. 
All these researches were carried out with enough information from the statistical data. Therefore, with a little information on population data, one finds it difficult to describe the process of population growth in statistics and other related fields. This therefore makes the application of the concept of algebraic topology which is abstract and requires fewer tasks which are very important in this study. Homological invariant in algebraic topology shortens this process and provides convenient techniques to the study of continuous process such as population growth. Indeed, homological invariants have proven to be a valuable tool for studying not only continuous process such as population growth but also for many continuous physical phenomena. For instance, Kinsey [3] used Euler characteristics as one of the topological invariants to study the surfaces of objects and concluded that the surface of an object is closed if the Euler characteristic is zero. This is one of the abstract techniques of describing a closed object. Hossenia et al. [4] also developed the concept of homotopy, one of the topological invariants via deformation and stabilization of the linear part of the homotopy equation. His method showed how the stability and convergence of a solution of equation was guaranteed. Many research works including others from $[4,5]$ which employed the concept of homological invariants were reviewed for this paper, but the major ideas of this paper were developed from the research works of Donald and Chang [5]. More often, the approach to the study of population growth is centered on statistics and differential equations $[1,6,7]$. In this paper, the novelty is the introduction of a homological invariant outside statistical and differential equations. The homological invariants we have introduced in this paper will describe the continuous paths between the 2-dimensional surfaces or groups and will be referred to as the growth of the population. In all cases, an increasing sequence of the paths of groups in the topological space is the population growth.

1.1. Background. In some previous works [6,7], statistical and differential equation methods were used to study the growth of populations from a given data set. To some extent, these statistical and differential equation models have proven to be successful methods in the study of both linear and nonlinear growth of population. Though there are other equally important methods which also attempt to establish the growth of population, none of these methods adopted the concept of groups in the algebraic category. The algebraic topology used the concept of the homological invariant which preserves certain topological structures of the 2-dimensional surface (algebraic category). The objects of the group are limited to only the 2-dimensional surface. The paths (continuous maps) are helpful in producing the extension. The extension of the groups by the continuous maps in this study is referred to as the population growth.

\section{Main Concept}

Population growth is developed from the objects of group and continuous maps. It occurs when one object is extended to another object by a path or line segment. By a path in population growth, we mean the continuous map. The population $(\eta)$ is a topological space $X$ generated by the objects of $v^{n}$.

\subsection{Preliminary Propositions}

Proposition 1. The population ( $\eta$ ) in a particular year is the $n$-dimensional surface or $n$-simplex $v_{i}^{n} \in X$ for $i=0,1,2,3, \ldots$.

Proposition 2. For any population ( $\eta)$, the growth of population occurs if

$$
H\left(\eta_{k}\right)=\left(v_{k+1}^{n}\right)-\left(v_{0}^{n}\right)=1,
$$

where $n=, 2,3, \ldots, k=1,2,3,4, \ldots$, and $v_{0}^{n} \in X$ is the initial $n$-dimensional surface.

Proof of Proposition 2. Let $\eta$ denote the population value in a given year and the change in the population be a homomorphism or distance $(d)$ from $v_{i}^{n} \in X$ to $v_{j}^{n} \in X$. In this case, $v_{j}^{n} \in X$ is the new population size from $v_{i}^{n} \in X$. Let $k \geq 1$ represent the years that growth occurs such that $k=1$ is the first year. At $k=1$, the population size from the initial population size $v_{0}^{n} \in X$ is $v_{k+1}^{n} \in X$. Let $\left\{v_{0}^{n}, v_{k+1}^{n}, \ldots\right\}$ and $\{d, 2 d, 3 d, \ldots\}$ represent the objects of population and their corresponding homomorphisms or distances such that $\left\{k=1=v_{2}^{n}=2 v^{2}=2, k=2=v_{3}^{n}=3 v^{3}=3, \ldots\right\} \quad$ and $\quad\{d=1,2$ $d=2,3 d=3, \ldots\}$. An increasing sequence of the individual objects of the space $X$ increases the size of the population. This is given as

$$
\begin{aligned}
& V=\sum_{\beta=1} \beta v^{n}, \\
& D=\sum_{\beta=1}(\beta-1) d .
\end{aligned}
$$

Therefore, the total growth in the population is given as

$$
H\left(\eta_{k}\right)=\sum_{\beta=1} \beta v^{n}-\sum_{\beta=1}(\beta-1) d,
$$

which is the same as

$$
H\left(\eta_{k}\right)=\sum_{\beta=1} v^{n}-\sum_{\beta=1} d .
$$

As the population grows from the first year to the second year, that is, $n=2$ and $k=1$, we have

$$
H\left(\eta_{2}\right)=\sum_{\beta=1}^{2} v^{n}-\sum_{\beta=1}^{1} d,
$$

which becomes

$$
H\left(\eta_{2}\right)=\left(v^{n}+v^{n}\right)-d=1 .
$$

Similarly, at $n=3$ and $k=3$, we have 


$$
\begin{aligned}
& H\left(\eta_{3}\right)=\sum_{\beta=1}^{3} v^{n}-\sum_{\beta=1}^{2} d, \\
& H\left(\eta_{3}\right)=\left(v^{n}+v^{n}+v^{3}\right)-(d+d)=1 .
\end{aligned}
$$

Therefore, at the $k$-th year, we have

$$
H\left(\eta_{k}\right)=\beta v^{n}-(\beta-1) d=1,
$$

which proves Proposition 2.

Proposition 3. Let $a<b$ such that the closed interval $[a, b] \in v_{i}^{n} \in X$ defines the size of the $i$-th population. Let also $c<d$ such that the closed interval $[c, d] \in v_{j}^{n} \in X$ where $[c, d]$ is larger than $[a, b]$. If $f$ is the homomorphism from $v_{i}^{n} \in X$ to $v_{j}^{n} \in X$ such that $f(a)=c$ and $f(b)=d$, then the growth of population occurs. Thus, for $[a, b]<[c, d],[c, d] \in v_{j}^{n} \in X$ and $[a, b] \in v_{i}^{n} \in X$, if $f:[a, b] \in v_{i}^{n} \longrightarrow[c, d] \in v_{j}^{n}$. Such that $f(a)=c$ and $f(b)=d$, and then the growth of population occurs. In terms of real values, population growth occurs when the final value of the previous population is the initial value of the current population.

2.2. Homology. Generally, the $n$th homology group of the topological space $X$ is defined as

$$
H_{n}=\frac{\operatorname{Kerd}_{n}}{\operatorname{Im} d_{n+1}}
$$

where $\operatorname{Kerd}_{n}$, the kernel of the boundary operator, $d_{n}$ represents the number of enclosed paths (cycles) in $X$, and the image $\left(\operatorname{Im} d_{n+1}\right)$ represents the edge (boundary) of the enclosed paths (cycles) in $X$. That is, $\operatorname{Im} d_{n+1} \subseteq \operatorname{Kerd}_{n}$. The topological space $X$ consists of a finite set of points $v_{i}^{2} \in X$ or 2 -dimensional surfaces for the population $\eta$, and the increase in population is the increase from one $v_{i}^{2} \in X$ to another. That is, $v_{i}^{2} \in X$ increases to $v_{j}^{2} \in X$ for $i<j$. Since $v_{i}^{2} \in X$ is a 2 -dimensional surface, zero, one, and two homology invariants can be estimated.

\subsection{Computation of $H_{0}$ and $H_{1}$ Homology Invariants}

2.3.1. Using 2-Dimensional Surface as the Simplicial Complex. In a situation where $v_{i}^{2} \in X$ is the 2-dimensional surface, the homology invariants are estimated as follows: If $v_{0}^{2} \in X$ is the initial population, and we assume that the $1^{\text {st }}$, $2^{\text {nd }}$, and $3^{\text {rd }}$ years have the following population values $v_{0}^{2}$, $2 v_{0}^{2}$, and $3 v_{0}^{2}$, then the zero-th homological invariant is estimated as

$$
H_{0}=\left(v_{0}^{n}, 2 v_{0}^{n}, 3 v_{0}^{n}, \ldots\right)=Z \text {, }
$$

where $Z$ is a constant and indicates the existence of an initial population.

If $v_{1}^{2} \in X$ is an increment of $v_{0}^{2} \in X$, then $v_{0}^{2} \in X \subset$ $v_{1}^{2} \in X$. However, for all $A \in v_{0}^{2} \in X$, KerA $\in v_{0}^{2} \in X$ and $\operatorname{Im} A \in v_{1}^{2} \in X$. Also, $\operatorname{Ker} M \in v_{1}^{2} \in X$ if $M \in v_{1}^{2} \in X$. Therefore, the first homology invariant is $H_{1}=\operatorname{Ker} M / \operatorname{Im} A$ since $\operatorname{Im} A \subset \operatorname{Ker} M$. From Lagrange's theorem, it can be deduced that the first homology invariant is $H_{1}=\operatorname{Ker} M / \operatorname{Im} A=\phi$ where $\phi \in Z$ is the extent of the increment.

2.3.2. Lagrange's Theorem. Let $G$ be a group and $H$ a subgroup of $G$, then in a finite group, the order of a subgroup divides the order of the group.

Proof. $G$ is a group and $H$ is a subgroup of $G . \forall \alpha, \beta \in G$, let the class of $\alpha$ be $[\alpha]$ such that for $k \in Z$, we have $k \alpha_{i}$ where $i=1,2, \ldots$ Consider $k \alpha_{i}$. If $k \alpha_{1}, k \alpha_{2}, k \alpha_{3}, \ldots, k \alpha_{n}$ are the $n$ number of distinct classes, then $G=\cup_{i=1}^{n} k \alpha_{i}$. Suppose the order of $k \alpha_{i}$ is $k$ denoted as $|k|$. If $G=\cup_{i=1}^{n} k \alpha_{i}$ and $k \alpha_{i}$ are disjoint, then $|G|=n|k|$, hence $|k|$ divides $|G|$.

\subsubsection{Using Differential Matrices from Smith Normal Form} $(S N F)$. Consider the 2-dimensional rectangular surfaces as shown in Figures 1 and 2, respectively.

Figure 1 is the initial population and has only one triangular plane in the rectangle such that the vertices are not repeated. Figure 2 is the enlargement of Figure 1 and also has two triangular planes in the new rectangle where all the vertices are different. $d_{1}$ and $d_{2}$ are the differentials in the initial and final populations. From Figures 1 and 2, the differential matrices $d_{1}$ and $d_{2}$ are given as

$$
\begin{aligned}
& (0,1) \quad(0,2) \quad(1,2) \quad(2,3) \quad(3,4) \quad(3,5) \quad(4,5) \\
& \left.d_{1}=\begin{array}{l}
\{0\} \\
\{1\} \\
\{3\} \\
\{4\} \\
\{5\} \\
1
\end{array}\right]\left[\begin{array}{ccccccc}
-1 & -1 & 0 & 0 & 0 & 0 & 0 \\
1 & 0 & -1 & 0 & 0 & 0 & 0 \\
0 & 1 & 1 & -1 & 0 & 0 & 0 \\
0 & 0 & 0 & 1 & -1 & -1 & 0 \\
0 & 0 & 0 & 0 & 1 & 0 & -1 \\
& 0 & 0 & 0 & 0 & -1 & -1
\end{array}\right] \\
& (0,1,2) \quad(3,4,5) \\
& \left.\left.\begin{array}{rl} 
& \{0,1\} \\
& \{0,2\} \\
& \{1,2\} \\
d_{2}= & \{2,3\} \\
& \{3,4\} \\
& \{3,5\} \\
& \{4,5\}
\end{array}\right] \begin{array}{cc}
1 & 0 \\
-1 & 0 \\
0 & 0 \\
0 & 1 \\
0 & -1
\end{array}\right]
\end{aligned}
$$

The Smith Normal Forms (SNF) from equations (13) and (14) are given in equations (15) and (16), respectively:

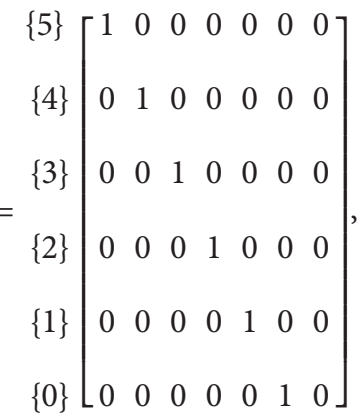




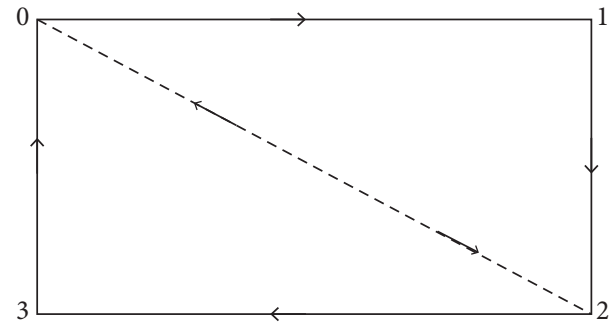

Figure 1: One 2-dimensional surface.

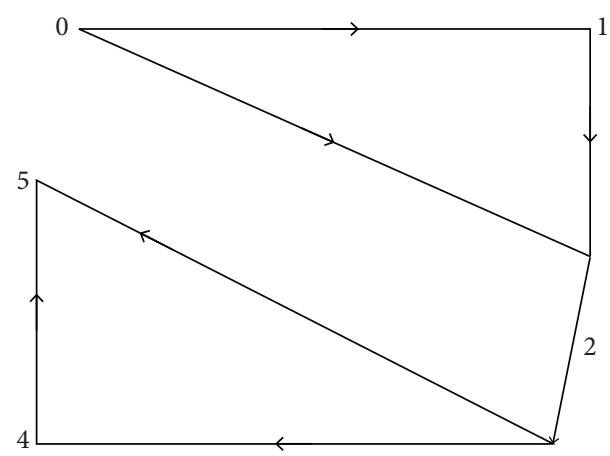

FIgURE 2: Two 2-dimensional surfaces.

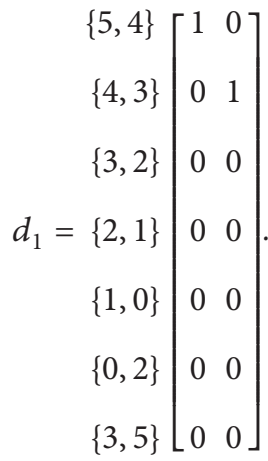

Using equation (15), $H_{0}=Z^{6-5-0}=Z$. Once again from equations (15) and (16), $H_{1}=Z^{7-2-5}=Z^{0}=1$ which confirms equation (12). Thus, $H_{0}=Z$ shows the existence of the initial population which grows with respect to time as indicated by $H_{1}=1$.

2.4. Topological Space from Data Set. $X=\mathfrak{R}^{2}$ is the topological space. Thus, the set $X$ together with the data points (years and the corresponding population values) as illustrated in Figure 1 is the topological space. A growth in population is an increasing sequence of the points, thus the 2-dimensional surface. With these points as the input, the homological invariants are identified.

Suppose $v_{i}^{2} \in X$ where $i=2,3,4, \ldots$ are the data points of the space $X$. From Figure $3, v_{i}^{2} \in X$ are connected together by paths $d\left(v_{i}^{2} v_{j}^{2}\right)$ for all $v_{i}^{2}, v_{j}^{2} \in X$. The addition of paths indicates the addition of given distances $d_{i}$ to $v_{i}^{2} \in X$ and hence providing the increasing sequence of $v_{i}^{2} \in X$ The growth of population is as a result of increasing sequence of

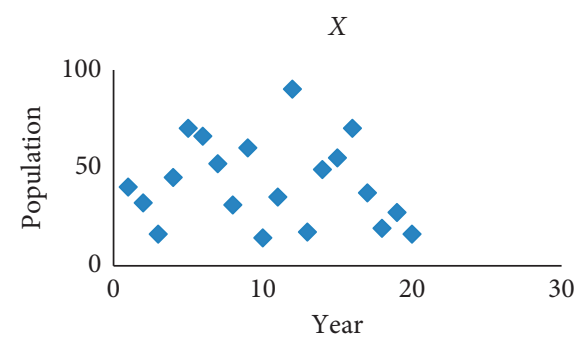

Figure 3: Topological space $X$ consisting of 2-dimensional surfaces.

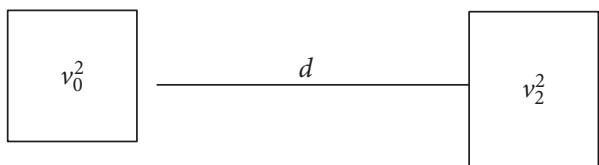

FIgURe 4: Change from the $1^{\text {st }}$ point to the $2^{\text {nd }}$ point.

$v_{i}^{2} \in X$ which is also determined by the $n$-th homology invariant $H_{n}=\operatorname{Kerd}_{n} / \operatorname{Im} d_{n+1}$.

\section{Homological Invariant and Population Growth}

From equation (1), it was assumed that the condition $H\left(\eta_{k}\right)=1$ for $k \geq 1$ must be satisfied to indicate an increase from one of $v_{i}^{2} \in X$ to another or to demonstrate growth in population $(\eta)$. However, the homological invariant $H\left(\eta_{k}\right)$ which determines the growth of population is defined as

$$
H\left(\eta_{k}\right)=\beta v^{n}-(\beta-1) d,
$$

where $\beta v_{i}^{2}$ and $(\beta-1) d$ denote the number of times the population increases and the extent to which population increases, respectively, for $\beta=2,3,4, \ldots$. From the first or initial data point to the second data point, there are two data points, that is, $n=2$ and $k=1$, as shown in Figure 4 .

Therefore, we have two $v_{i}^{2} \in X$ and only one distance $(2-1) d$ between the two $v_{i}^{2} \in X$. Hence, the $1^{\text {st }}$ homological invariant is

$$
H\left(\eta_{1}\right)=2-1=1
$$

The homological invariant $H\left(\eta_{k}\right)=1$ indicates that there is a growth in the population from the first-year data point to the second-year data point. Similarly, from the first data point to the fourth data point, there are four $v_{i}^{2} \in X$ with three distances between them, thus $\beta=4$ and $k=3$. The $3^{\text {rd }}$ homological invariant from the first-year data point to the fourth-year data point is

$$
H\left(\eta_{3}\right)=4-3=1 \text {. }
$$

The homological invariant $H\left(\eta_{3}\right)=1$ is an indication that there is a growth in population from the first to the fourth year.

\section{Conclusion}

The paper constructed and defined the homological invariant as an efficient tool to analyse the growth of the 
population. The topological information from a geometric object was applied to a data set from statistics in a 2-dimensional Euclidean plane as shown in Figure 3 to obtain the information about the population growth. It was observed that the homological invariant offers another reliable technique to handle information regarding the population growth or the extension of the data set. An advantage of this method over the statistical and differential equation methods is that it simply explains the growth in the population using the time value and the homological invariant. In this work, it was assumed that anytime $v_{i}^{2} \in X$ exceeds the path by one, extension occurs. By extension, we mean the changes in population values and this was determined using the homological invariant. Therefore, whenever the homological invariant condition $H\left(\eta_{k}\right)=1$ is satisfied as shown in equations (18) and (19), there is a growth in the population.

The homological invariant method offered new important insights and information about the growth of population. The different methods of computing the homological invariant $H(\eta)$ with known $v_{i}^{2} \in X$ were presented. The methods were credible and simple. Its approach requires less information and was very simple when it was compared with statistics and differential equation approaches. The paper established a homological invariant to show that every population in a topological space grows. It is believed that the analysis of the population growth using a homological invariant within the algebraic topology concept is a contribution to knowledge. The most important result of this paper is the following proposition.

Proposition 4. For any population ( $\eta)$, the growth of population occurs if

$$
H\left(\eta_{k}\right)=\left(v^{n}{ }_{k+1}\right)-\left(v_{0}^{n}\right)=1 .
$$

\section{Data Availability}

No data were used to support this study.

\section{Conflicts of Interest}

The authors declare no potential conflicts of interest with respect to the research, authorship, and publication of this paper.

\section{References}

[1] R. J. Walker, "Population growth and its implications for global security," American Journal of Economics and Sociology, vol. 75, no. 4, 2016.

[2] T. Guney, "Population growth and sustainable development in developed-developing countries, an IV (2SLS) approach," The Journal of Faculty of Economics and Administrative Science, vol. 22, no. 4, pp. 1255-1277, 2017.

[3] C. L. Kinsey, Topological of Surfaces, Springer-Verlag, New York, NY, USA, 1993.

[4] S. H. Hossenia, A. Ranjar, and S. Momani, "Using an enhanced homotopy perturbation method in fractional differential equations via deforming the linear part," Computers \&
Mathematics with Applications, vol. 56, no. 12, pp. 3138-3149, 2008.

[5] B. R. Donald and D. R. Chang, "On the complexity of computing the homology type of triangulation," in Proceedings of the 32nd Annual Symposium of Foundations of Computer Science, pp. 650-661, San Juan, PR, USA, October 1991.

[6] B. S. T. Alkahtani, A. Atangana, and L. Koca, "New nonlinear model of population growth," PLoS One, vol. 12, no. 10, Article ID e0184728, 2017.

[7] F. W. E. Peterson, "The role of population in economic growth,” pp. 1-15, 2017, http://Journals.sagepub.com/home/ sgo.

[8] E. R. Lenski and M. P. Service, "The statistical analysis of population growth rates calculated from schedule of survivorship and fecundity," Ecological Society of American Journal, vol. 63, no. 3, pp. 655-662, 1982. 